\section{Microbiological dictionary}

Dictionary of Microbiology. By $\mathbf{P}$. Singleton and D. Sainsbury. Pp. 481. (Wiley: Chichester, UK, and New York, 1978.) $£ 17.50$.

IT seems to me that two sorts of useful dictionary could be compiled for microbiology. The truly comprehensive dictionary would be a large scholarly work and probably could only be written by five or six people. But to have a single reference work for the whole terminology of this subject (stretching from phytoplankton ecology to the molecular biology of animal viruses) would be of enormous value. The second sort of dictionary is not comprehensive and is of little use to the professional microbiologist but can be of great value to those who lack a microbiological background yet who need to know about some aspects of microbiology in association with another main interest.

The authors' preface indicates that this dictionary is intended for undergraduates and postgraduates in microbiology but I think it is only likely to find a place in my second category. Microbiology students would find little

\section{Steady-State \\ Enzyme Kinetics}

\section{Stanley \\ Ainsworth}

Identifies the basic ideas of the subject for the graduate and advanced undergraduate student, and

familiarises the reader with them so that they may then be applied to any problem likely to be encountered.

Although it cannot altogether avoid mathematical presentation, almost all the equations used require only an elementary acquaintance with algebraic manipulation. Moreover the arguments themselves are presented as fully as possible to enable the reader to progress easily fron each stage to the next.

$£ 10.000333150082$

To obtain a copy of this book, please order through your normal supplier or send $₹ 11.00$, including carriage to

Technical Books, Services, P.O. Box 79, Maidenhead, Berkshire SL6 2EG.

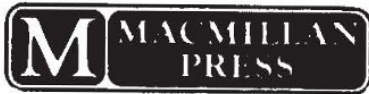

in the dictionary which was not covered at least as well by their textbooks, even if they have only a very small collection. The complete absence of any references to source of information or to more detailed literature would also reduce the use which could be made of the contents.

The authors have set out to cover "pure and applied microbiology, biochemistry, immunology, genetics and microbiological aspects of allied subjects such as medicine, veterinary science and plant pathology". This is probably too wide a range of material to encompass in less than 500 pages and has led to omissions. Although I was not surprised that there is no entry for Sterigmatomyces, it is unfortunate that for instance Trichoderma and Dunaliella are not included; that is, the choice of organisms has been rather arbitrary, with some very obscure genera included and some important or commonly used ones left out. Comparable examples are the absence of bacteriophages $\mathrm{mu}$ and $\mathbf{P 1}$, which have an important place in microbial genetics.

The coverage of biochemical terms may equally be criticised for the lack of entries concerning microbial products that are of interest in a wider context, such as pronase, subtilisin and taka diastase. A much fuller account of restriction endonucleases would also have been a timely improvement.

The cross reference of one entry to another is not thorough. For instance, although the entry "red tides" attributes this phenomenon to the alga Gonyaulax, the entry "Gonyaulax"

\section{Basic phycology}

IN the introduction to Introductory Phycology (Wiley: Chichester, UK; $£ 12.25$ ), F. R. Trainor stresses the importance of consulting more specific works in order to learn how new information is added (to the science) and older facts and interpretations are modified or discarded. Such an outlook was clearly foremost in the author's mind when writing this book for throughout the book the different subjects are suitably placed in their historical perspective and questions are raised in areas deserving of attention in a way that a student might identify with the specialist researcher. The book is perhaps suitable not so much as a reference text but to be read straight through as an introduction to the subject. As such it is well conceived. The author succeeds in conveying his interest and enthusiasm with style and the layout has obviously been makes no mention of red tides. Similarly, although the Helmstetter-Cooper model of the bacterial chromosome replication cycle is described at some length under "growth (bacterial)", the entry "Cell cycle" does not refer to it. Indeed, the entry for "Cell cycle" gave me the clear impression that this term is only used with reference to eukaryotic (usually mammalian) cells, which is misleading.

Coverage of growth physiology and fermentation is poor, with a wide variety of omissions. There is no mention of substrate-accelerated death, maintenance energy or fed-batch culture, which are the kind of terms which non-microbiologists are most likely to seek for definition in a subject dictionary of this sort. The description of continuous culture is very sketchy.

An appendix of metabolic pathway schemes is included, which is a laudable idea, but again the selection is not beyond criticism. Why the tricarboxylic acid cycle is in this appendix whereas the reductive pentose phosphate cycle is in the main body of the text (under "Photosynthesis") is not obvious.

Recognising the limitations of space I feel that many will share the view that too much space has been devoted to material which can easily be found in the literature of biochemistry, genetics and general biology at the expense of many microbiological terms and ideas which could so usefully have been included. Christopher F. Thurston

Christopher $F$. Thurston is Lecturer in Microbiology at Queen Elizabeth College, University of London, UK.

given a good deal of consideration.

Following two introductory sections each of the algal groups is considered separately in twelve chapters, which should give the student a fairly comprehensive account of algal forms. In each of these Trainor deals with the morphological organisation, the characterisation and the reproduction of the class before considering representative genera and other features of special interest. The classification of each group has been deliberately kept to a minimum. Although this is laudable in many respects it might serve to confuse a student particularly in a group like the Chlorophyceae.

The succeeding chapters deal with algal habitats and distribution, classification and morphological variability of benthic organisms, the plankton and their environment, algal control, their usefulness and harmful effects, and finally their culture. In the course of these a good deal of interest is generated and many aspects are treated in 